\title{
Modulation Excitation PM-IRRAS: A New Possibility for Simultaneous Monitoring of Surface and Gas Species and Surface Properties
}

\author{
Atsushi Urakawa§a ${ }^{\S}$ Thomas Bürgi ${ }^{b}$, and Alfons Baiker ${ }^{\star a}$ \\ §SCS Poster Prize Winner
}

\begin{abstract}
Polarization-Modulation Infrared Reflection-Absorption Spectroscopy (PM-IRRAS) is a sensitive tool for the analysis of species residing at gas-solid and gas-liquid interfaces. The polarization-modulation allows excellent back-ground compensation and the analysis of surface/interface species under moderate pressure (e.g. atmospheric pressure of IR-absorbing gases) is possible. We demonstrate a new possibility to extract simultaneously information of gas and solid phases in addition to surface species from the PM-IRRAS experiments, using CO oxidation over Pt film as an example. Modulation Excitation Spectroscopy (MES) has been combined with this technique to enhance the sensitivity and to analyze the kinetic behavior of species. The surface species involved in the oxidation process, the state of $\mathrm{Pt}$, and the gas phase species $\left(\mathrm{CO}\right.$ and $\left.\mathrm{CO}_{2}\right)$ could be simultaneously monitored in situ and analyzed quantitatively. The technique can serve as a valuable tool for investigations of various dynamic phenomena occurring at gas-solid interfaces.
\end{abstract}

Keywords: CO oxidation · Gas-solid interface · In situ spectroscopy · Modulation Excitation Spectroscopy · PM-IRRAS

\section{Introduction}

Numerous chemical and physical processes occur on surfaces, such as adsorption, desorption, reaction, and surface reconstruction. Detection of such surface processes and of the species involved has been and will be a big challenge in surface science, catalysis, and material sciences. Nowadays, it is a well-known fact that most of practically relevant surface processes only occur

\footnotetext{
Correspondence: Prof. Dr. A. Baikera

Tel.: +4144632 3153

Fax: + 41446321163

E-Mail: baiker@chem.ethz.ch

aETH Zürich

Institute for Chemical and Bioengineering

Department of Chemistry and Applied Biosciences

$\mathrm{CH}-8093$ Zürich

bUniversité de Neuchâtel

Institut de Chimie, Faculté des Sciences

Rue Emile-Argand 11

$\mathrm{CH}-2007$ Neuchâtel
}

under realistic conditions, in other words, using non-simplified material under technologically relevant pressure. Traditionally, the use of ultra-high vacuum (UHV) conditions and single crystals has been the method to monitor surface processes in order to minimize the effects of the liquid or gas phase above the surface. Although rich information can be obtained by UHV studies with single crystals, the relevance of the information to the industrial processes is often doubted. In fact, recent studies on $\mathrm{CO}$ oxidation under realistic conditions revealed a new phase, species, and dynamic behavior [1][2]. This is the main reason why many researchers now attempt to monitor surface processes under realistic conditions aiming at 'bridging the material and pressure gap'.

Polarization-modulation infrared reflection-absorption spectroscopy (PM-IRRAS) [3] and sum frequency generation (SFG) [4] are popular surface-sensitive techniques in vibrational spectroscopy used to "bridge the gap'. In the present work, the application of PM-IRRAS has been developed further to monitor not only surface but also gas phase species. In addition to the information about species in these two phases, surface proper- ties, such as surface phase changes, can be extracted from the reflectivity change.

\section{IRRAS}

The electric field in an unpolarized light beam can be mathematically separated into two mutually orthogonal components, $p$ and $s$-polarizations. For $p$-polarization the electric field vector is oscillating parallel to the plane of incidence as illustrated in Fig. 1. Similarly, the $s$-polarized light has the oscillation of its electric field perpendicular to the plane of incidence. When a light is reflected from a surface, a phase shift occurs between the incident and reflected light. According to the Fresnel equations [5] for a metallic surface, $s$-polarization undergoes a phase change of $\mathrm{ca} .180^{\circ}$ at all angles of incidence, resulting in destructive interference and no net electric field near the surface (Fig. 1). On the other hand, little phase change occurs for $p$-polarization except under extremely grazing conditions (angle of incidence (Fig. 1) $\theta_{i}>80^{\circ}$ ) and the interference becomes constructive. Therefore, the z-component of the electric field is enhanced (Fig. 1) and the enhancement is maximized near grazing angle due to the large $\mathrm{z}$-component of the $p$-polarization. In 

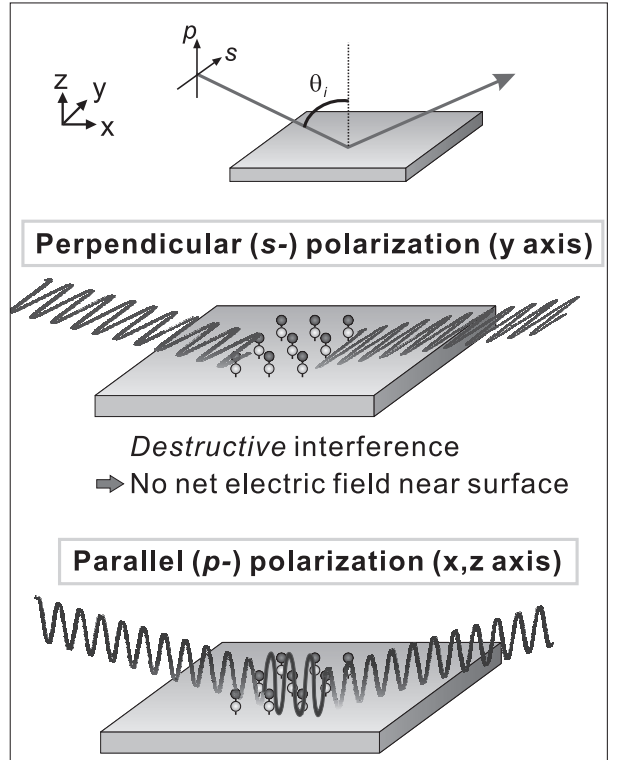

Constructive interference (z axis) $\Rightarrow$ Enhanced electric field near surface

Fig. 1. Definition of $s$ - and $p$-polarization, angle of incidence $\theta_{i}$, and schematic drawing of IRRAS principle

infrared reflection-absorption spectroscopy (IRRAS) often only the $p$-polarization is used to detect surface species typically under UHV conditions.

\section{PM-IRRAS and the Idea Behind this Work}

PM-IRRAS uses both $p$ - and $s$-polarizations. The two polarizations are alternatingly generated by a photoelastic modulator operating at a high frequency $(\sim 50-100$ $\mathrm{kHz}$ ) and the reflectivities of both polarizations, $\mathrm{R}_{\mathrm{p}}$ and $\mathrm{R}_{\mathrm{s}}$ (Fig. 2), are detected. Fig. 2 shows the principle of PM-IRRAS and the idea behind the current study. When IR light is reflected off a metallic surface in the presence of gas phase and surface species, $\mathrm{R}_{\mathrm{s}}$ contains only gas phase information due to the lack of surface electric field of the $s$-component, while $\mathrm{R}_{\mathrm{p}}$ contains both gas phase and surface information. Clearly, the difference between $s$ - and $p$-reflectivities, $\Delta \mathrm{R}=\mathrm{R}_{\mathrm{p}}-\mathrm{R}_{\mathrm{s}}$, yields surface species information and the ratio between the difference and sum reflectivity $\Delta R / R\left(R=R_{p}+R_{s}\right)$ is used to compensate the gas phase absorbance, hence yielding a PM-IRRA surface spectrum. Conventionally, $\mathrm{R}_{\mathrm{S}}$ is only used to compensate the bulk phase contribution (gas or liquid phase above a surface), resulting in excellent signal to noise ratio thus allowing fast time-resolved measurements (order of seconds). In this work, we made use of $R_{S}$ to study the evolution of gas phase species in addition to the conventional use of PM-IRRAS, i.e. the study of surface species. It should be emphasized that both surface and gas phase information can be obtained simultaneously. The technique was combined with modulation excitation spectroscopy (MES) [6] to further enhance the sensitivity and to accurately study the dynamic behavior of surface processes with a good time resolution. CO oxidation over a Pt film was studied and the potential and sensitivity of the technique, modulation excitation (ME) PM-IRRAS, are demonstrated.

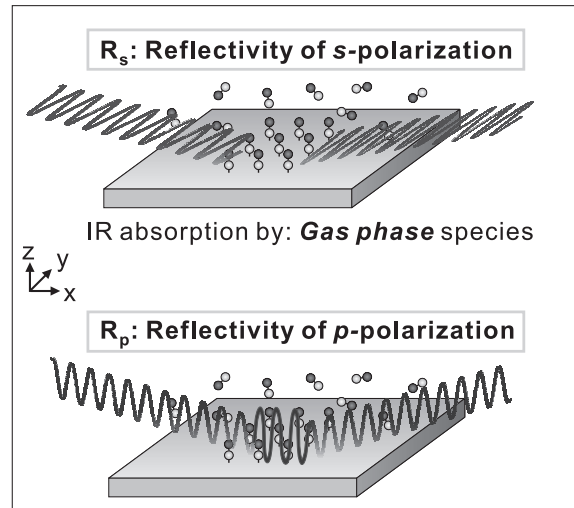

IR absorption by: Gas phase + surface species

$\left(\mathbf{R}_{\mathrm{p}}-\mathbf{R}_{\mathrm{s}}\right) \sim$ Surface species

$\mathbf{R}_{\mathrm{s}}$ or $\left(\mathbf{R}_{\mathrm{p}}+\mathbf{R}_{\mathrm{s}}\right) \sim$ Gas phase species

Fig. 2. Schematic drawing of PM-IRRAS principle for the simultaneous detection of surface and gas species

\section{Experimental}

A small-volume cell for ME PM-IRRAS with a short light path length was designed [7]. The gas mixing property within the cell is excellent and fast exchange of gas species is possible [8]. Pt film was used to study $\mathrm{CO}$ oxidation. An aluminum plate was coated with $40 \mathrm{~nm}$ Pt by means of physical vapor deposition and the sample was mounted in the cell located within the compartment of a Bruker PMA 37, connected to the external beam port of a vector 33 Fourier transform infrared spectrometer. The angle of incidence was $80^{\circ}$. CO and $\mathrm{O}_{2}$ gas, both in $\mathrm{He}$, were alternatingly passed into the cell (concentration modulation) and the obtained spectra were averaged over a number of modulation periods to enhance the signal-to-noise ratio. The obtained time-domain spectra were $d e$ modulated by a mathematical treatment, a so-called phase sensitive detection (PSD). The obtained spectra after PSD are in the phase-domain and all the frequencies of the response except one (the demodulation frequency) are filtered out, leading to significant sensitivity enhancement and kinetic differentiation [6][9]. The details of the cell, experiments, and mathematical treatments of spectra can be found elsewhere [7].

\section{Results and Discussion}

\section{Time-Domain Analysis}

Fig. 3 shows the PM-IRRA surface and gas spectra of a $\mathrm{CO} / \mathrm{O}_{2}$ modulation experiment under atmospheric pressure at 433 $\mathrm{K}$. An adsorbed atop $\mathrm{CO}$ band was clearly observed at $2100 \mathrm{~cm}^{-1}$ under the $\mathrm{CO}$ atmosphere, and the $\mathrm{CO}$ band sharply and completely disappeared a while (ca. $20 \mathrm{~s}$ ) after the gas was switched to oxygen, accompanied by a broadband step increase of the baseline. When the gas was switched to $\mathrm{CO}$, a sharp increase in the adsorbed $\mathrm{CO}$ band was observed with a step decrease of the baseline. The broadband baseline move was explained by the change in the refractive index of near-surface bulk, i.e. from $\mathrm{Pt}$ to Pt oxide and vice versa [7]. This surface reconstruction likely plays a key role for the rapid increase in the adsorbed $\mathrm{CO}$ by formation of metallic Pt surface under $\mathrm{CO}$ atmosphere.

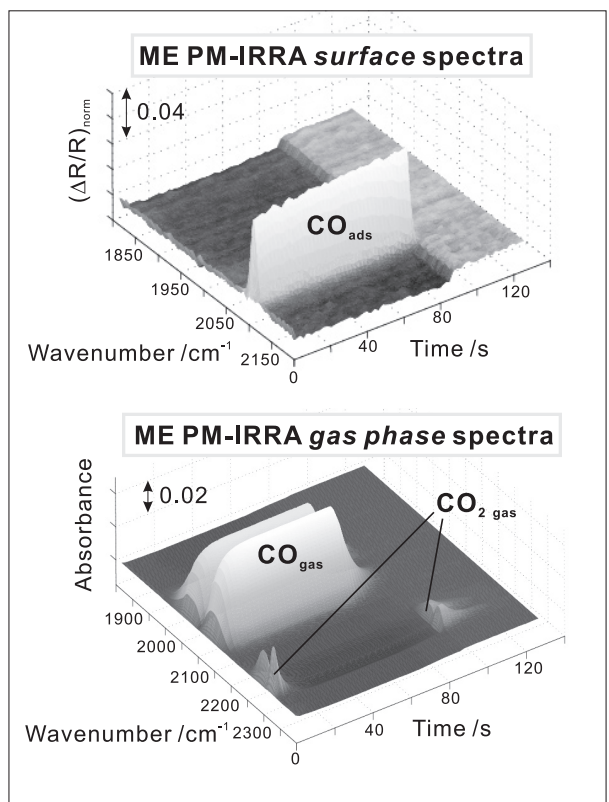

Fig. 3. Modulation excitation PM-IRRA surface and gas phase spectra of $\mathrm{CO}$ and $\mathrm{CO}_{2}$ stretching frequency region. 1st half period: $5 \% \mathrm{CO}$ in $\mathrm{He}$, 2nd half period: $40 \% \mathrm{O}_{2}$ in $\mathrm{He}$, both $60 \mathrm{ml} / \mathrm{min}$, modulation period: $128 \mathrm{~s}$, at $433 \mathrm{~K}$.

The oxidation product, gaseous $\mathrm{CO}_{2}$, could be detected; it was formed when the gas was switched to $\mathrm{CO}$ and during the exposure to $\mathrm{O}_{2}$. Effects of $\mathrm{O}_{2}$ concentration on the reaction were analyzed and the temporal profiles of the adsorbed $\mathrm{CO}$ and gaseous $\mathrm{CO}_{2}$ are shown in Fig. 4. The responses of $40 \% \mathrm{O}_{2}$ in Fig. 4 correspond to the results shown in Fig. 3. Under $\mathrm{CO}$ atmosphere after the gas exchange, instantaneous formation of $\mathrm{CO}_{2}$ was observed (Fig. 4 (B)). The $\mathrm{CO}_{2}$ concentration under $\mathrm{CO}$ atmosphere decreased according to the mixing property of the cell, meaning that all $\mathrm{CO}_{2}$ was formed at once (Fig. 4 (B)). In contrast, two 


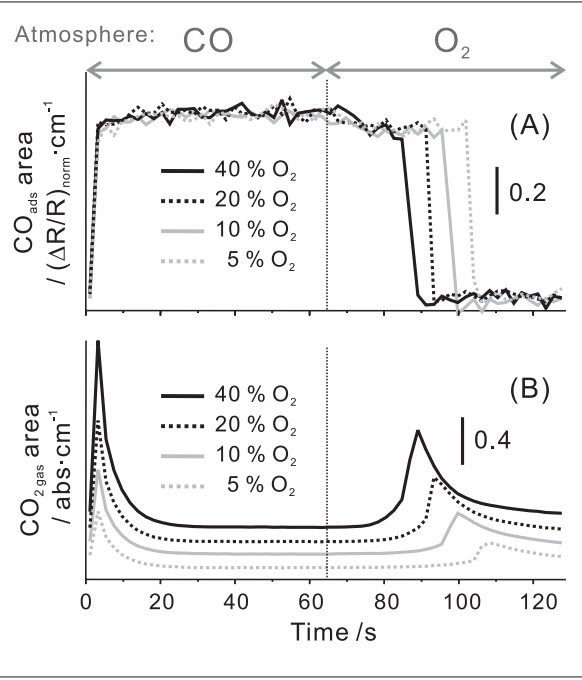

Fig. 4. Concentration profiles of (A) adsorbed $\mathrm{CO}$ and $(\mathrm{B})$ gaseous $\mathrm{CO}_{2}$ during the modulation experiment. Experimental conditions are the same as those described in Fig. 3 except $\mathrm{O}_{2}$ concentration.

reactive phases, characterized as low and high activity phase, respectively, were observed under $\mathrm{O}_{2}$ atmosphere (Fig. 4 (B)). The transition between the active phases coincided with the baseline step change, indicating that the highly active surface is surface Pt oxide. A large amount of atop $\mathrm{CO}$ was observed during the low activity phase, whereas no atop $\mathrm{CO}$ was observed during the high activity phase (Fig. 4 (A)). The tail behavior of the $\mathrm{CO}_{2}$ formation under $\mathrm{O}_{2}$ atmosphere was different from the one under $\mathrm{CO}$ atmosphere, not following the mixing property of the cell and not reaching zero concentration at the end of the period. This is a clear indication that the reaction mechanisms under $\mathrm{CO}$ and $\mathrm{O}_{2}$ atmospheres are different. The induction time of the transition from the low to the high activity phase was $\mathrm{O}_{2}$ concentration dependent (Fig. 4). Further analysis indicated that the high activity phase is formed only below a certain threshold $\mathrm{CO}$ concentration under $\mathrm{O}_{2}$ atmosphere, more precisely below a threshold $\mathrm{CO} / \mathrm{O}_{2}$ ratio [7]. The experiments under various conditions (different temperatures and modulation frequencies) and the quantitative analysis suggested the possible origin of the $\mathrm{CO}_{2}$ formation under $\mathrm{CO}$ and $\mathrm{O}_{2}$ atmosphere [7]. The presence of slowly desorbing $\mathrm{CO}_{2}$ precursor in the $\mathrm{O}_{2}$ atmosphere was indicated. The latter desorbed at once when the gas phase was switched to $\mathrm{CO}$ atmosphere, triggered by the reconstruction of the surface Pt phase and strong adsorption of $\mathrm{CO}$ on metallic Pt. All the observations of this study are in excellent accordance with a transient high-pressure STM study [10] and complement the molecular insights gained into the reaction mechanism of $\mathrm{CO}$ oxidation over Pt.

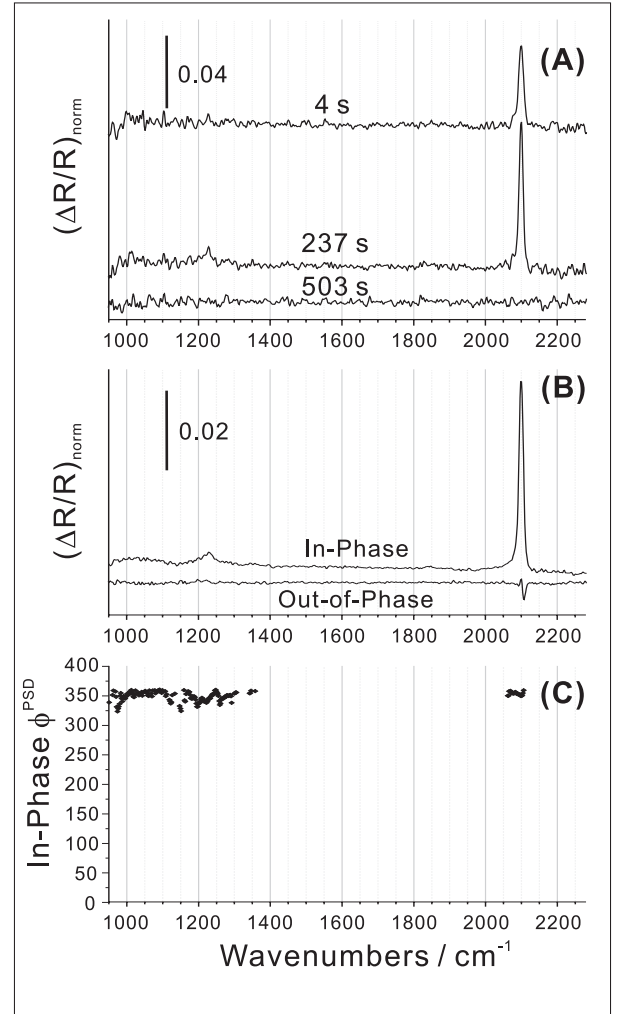

Fig. 5. (A) Selected time-domain spectra at different time during the modulation period, $(\mathrm{B})$ In-phase $\left(\phi^{\mathrm{PSD}}=349^{\circ}\right)$ and out-of-phase $\left(\phi^{\mathrm{PSD}}\right.$ $\left.=259^{\circ}\right)$ phase-domain spectra, and (C) In-phase angles of the phase-domain spectra. 1st half period: $5 \% \mathrm{CO}$, 2nd half period: $40 \% \mathrm{O}_{2}$, both in $\mathrm{He}$ at $60 \mathrm{ml} / \mathrm{min}, \mathrm{T}=516.1 \mathrm{~s}$ at $433 \mathrm{~K}$. 'In-Phase' and 'Out-of-Phase' angle are the phase angles $\phi^{P S D}$ at which amplitude in the phase domain are maximized and minimized, respectively (see [6] for details). For (C), the in-phase angles are shown for angles with in-phase amplitudes above a cutoff of 0.001 (in normalized IRRA unit).

\section{Phase-Domain Analysis}

The power of MES is the sensitivity enhancement and the possibility of kinetic analysis of phase-domain spectra obtained via the PSD of time-domain spectra. Fig. 5 shows the time-domain and the phase-domain PM-IRRA surface spectra of a $\mathrm{CO} / \mathrm{O}_{2}$ $\mathrm{ME}$ experiment. The time-domain spectra at three different points of the modulation period are noisy and it is difficult to analyze surface species except adsorbed CO. On the other hand, the signal to noise ratio is significantly improved in the phase-domain spectra. Two other bands (1050 and $1230 \mathrm{~cm}^{-1}$ ) were clearly observed besides the adsorbed CO $\left(2100 \mathrm{~cm}^{-1}\right)$. The in-phase angles, containing the kinetic information of species, were the same for all the bands, showing that the dynamic behavior, appearance and disappearance, of the species is the same. Ex-situ PM-IRRAS analysis after a series of modulation experiments showed that the two bands increased and merged into a broad peak [7] indicating an accumulation of a contaminant on the surface produced during reaction. The $\mathrm{CO}_{2}$ precursor suggested before is most likely infrared-inactive since the species absorbing at 1050 and $1230 \mathrm{~cm}^{-1}$ do not show the kinetic behavior expected for the precursor. A possible precursor is simply $\mathrm{CO}_{2}$ adsorbed parallel to the surface, thereby not visible in IR.

\section{Conclusions}

The potential of ME PM-IRRAS has been successfully demonstrated by simultaneous monitoring of surface and gas species under semi-realistic conditions (high temperature and pressure) in $\mathrm{CO}$ oxidation over the Pt film with good time resolution and high sensitivity. The simultaneous surface and gas phase measurements resulted in rich information leading to a rather complete picture of the reaction system. The combination of PM-IRRAS with ME technique allows the dynamic behavior of surface and gas phase species to be studied with high sensitivity. ME PM-IRRAS can serve as a valuable tool for investigations of various phenomena occurring at gas-solid interfaces.

\section{Acknowledgements}

Financial support by Foundation Claude and Giuliana is gratefully acknowledged.

Received: February 19, 2006

[1] G.A. Somorjai, K.S. Hwang, J.S. Parker, Topics in Catalysis 2003, 26, 87.

[2] H. Over, M. Muhler, Prog. Surf. Sci. 2003 72,3 .

[3] T. Buffeteau, B. Desbat, J.M. Turlet, Appl. Spectrosc. 1991, 45, 380.

[4] Y.R. Shen, Nature 1989, 337, 519.

[5] J.D. McIntyre, D.E. Aspnes, Surf. Sci. 1971, 24, 417.

[6] D. Baurecht, U.P. Fringeli, Rev. Sci. Instrum. 2001, 72, 3782.

[7] A. Urakawa, T. Bürgi, H.-P. Schläpfer, A. Baiker, J. Chem. Phys. 2006, 124, 054717.

[8] A. Urakawa, T. Bürgi, A. Baiker, Chem. Phys. in press.

[9] T. Bürgi, A. Baiker, J. Phys. Chem. B 2002, 106, 10649.

[10] B.L.M. Hendriksen, J.W.M. Frenken, Phys. Rev. Lett. 2002, 89, 046101. 